\title{
Current concepts and unmet needs in psoriatic arthritis
}

Dr Farrouq Mahmood ${ }^{1}$, Clinical Research Fellow in Rheumatology, Bradford \& Leeds Teaching Hospitals, United Kingdom

Dr Laura Coates ${ }^{2}$, NIHR Clinician Scientist, University of Oxford and Honorary Consultant Rheumatologist, Oxford University Hospitals, Oxford, UK

Dr Philip Helliwell ${ }^{3}$, Senior Lecturer \& Honorary Consultant in Rheumatology, Bradford \& Leeds Teaching Hospitals, United Kingdom Word count 4616 (excluding abstract, table and references) Abstract word count 133

Keywords: Enthesitis, Biomarkers, Classification, CASPAR, Treatment

Please send correspondence to:

Dr Philip Helliwell, Leeds Institute of Rheumatic and Musculoskeletal Medicine, University of Leeds, Chapel Allerton Hospital, Chapeltown Road, Leeds, LS7 4SA, UK.

Email: P.Helliwell@leeds.ac.uk 


\section{Abstract}

Psoriatic Arthritis is a chronic inflammatory arthritis that is part of the spondylarthropathy group of rheumatic diseases and has associated co-morbidities. It can present with various clinical manifestations making diagnosis and treatment challenging, resulting in significant disability and reduced quality of life for patients. While there have been advances in understanding the pathogenic mechanisms of the disease which have resulted in targeted therapies, there is still the need for further studies as some patients fail or are intolerant of current therapies. Better identification of early disease and knowledge of prognostic markers would enable clinicians to initiate appropriate therapy with the expectation that early aggressive treatment will minimise joint damage progression. Improved knowledge of the condition would also enable clinicians to better tailor specific treatment strategies for each of the various clinical domains in psoriatic arthritis. 
Introduction

Psoriatic Arthritis (PsA) is a chronic auto-immune inflammatory arthritis associated with psoriasis. Once thought to be a relatively mild disease, there has been an increasing recognition that PsA can result in significant disease burden for patients and healthcare services [1]. It can lead to severe disability and pain for the affected individual, and can negatively impact on daily function [2]. The disease burden for patients is comparable to other serious pathologies such as cancer [2].

Observational studies suggest early diagnosis is crucial in order to initiate treatment to minimise progressive joint damage. This can be challenging especially for non-specialists as PsA can present in myriad of ways and can be misdiagnosed for another condition such as gout [1]. No validated biomarkers or diagnostic criteria are yet available to aid clinicians in making a diagnosis [3], unlike in rheumatoid arthritis. One study showed an average diagnostic delay of five years between a patient presenting with joint pains and finally being diagnosed with PsA [4]. Under-diagnosis of PsA remains a problem in clinical care [5] and better screening tools are needed.

Management of the condition can often be equally as difficult. Effective treatment often requires multi-disciplinary collaboration as both the skin and joints need to be adequately treated. Dermatologists and Rheumatologists may have different approaches to initiating and using biologic therapy which could result in patients being undertreated [5]. Concerns over long term safety profiles of biologics may also limit use by clinicians [5]. Patients can find the treatments available burdensome due to side effects or constant blood monitoring, leading to poor compliance and dissatisfaction [4]. PsA is a complex disease and its management can present a significant challenge to the clinician and patient, especially if a patient has already failed multiple disease modifying drugs and biologic therapy, limiting the amount of therapeutic options available. 
Although there has been significant progress made in the understanding of PsA, gaps still remain in effective diagnosis and treatment which need addressing in future research. Such questions might include:

- Are there any genetic or bio markers for severity or treatment response?

- Are current treatment strategies adequate?

- Are there any advances in treatment which could be applied to PsA?

This article will explore current understanding of PsA and will highlight some of the unmet needs that need addressing.

\section{Pathogenesis}

PsA is a highly hereditable condition. The recurrence ratio of PsA among first degree relatives is higher than in psoriasis and is estimated at 27[6]. PsA has greater hereditability than other rheumatic diseases such as rheumatoid arthritis and Sjogrens syndrome [7]. Studies have shown that patients with a positive family history of psoriatic arthritis are at increased risk of developing the same condition themselves [8], with a positive family history of psoriasis having less influence. Up to $30 \%$ of psoriasis patients develop PsA. Why only $30 \%$ are affected remains unclear. Although PsA and psoriasis share some genetic overlap, there are also genetic differences between the two conditions [2]. These differences in association with certain environmental stimuli may be the reason why some patients develop PsA over others [2]. What exactly triggers PsA though remains unknown. A viral aetiology has been suggested although results from studies have been conflicting [9]. If genetic risk factors and environmental stimuli can be accurately identified for PsA then clinicians may be able to predict exactly which patients may develop the disease, and which patients may respond more favourably to certain treatments thus enabling more targeted treatment selection [7] and avoiding treatment failure. Researchers are also attempting to identify if and how having a 
particular geneotype may lead to developing a distinct clinical phenotype such as predominantly axial disease [10], which again would help clinicians select the most appropriate treatment for that particular phenotype. Recent studies in this area have identified certain HLA susceptibility genes which have been associated with particular PsA phenotypes [11]. There is also ongoing work on developing biomarkers to help identify early PsA and predict treatment response [12].

The exact pathogenic mechanism behind PsA is still unclear; however there have been several theories that have been proposed. One hypothesis is that PsA acts very much like a classic autoimmune disease, with an inflammatory cascade triggered by CD8 T cells binding to self-peptides through major histocompatibility complex (MHC) class 1 molecules [13]. CD8 T cells are found in abundance in synovial tissue in PsA patients, compared with CD4 cells in RA patients [10]. Further supporting this theory is the observation made in HIV patients of severe Pso and PsA occurring when there is CD4+ $t$ cell depletion, thus suggesting that PsA is CD8 driven [10]. CD8 cells are inappropriately activated due to a constant supply of self-peptides, thus eventually leading to inflammation. In addition peripheral blood of PsA patients show osteoclast progenitors which could explain the bone lesions that are observed [13]. Bony proliferation which is a hallmark of PsA may be driven by interleukin-22 by activating osteoblasts [3].

Another hypothesis is that the spondyloarthropathy group of diseases, which PsA belongs to, are driven by pro- inflammatory cytokines released by entheseal tissue. The enthesis may be the primary site of inflammation in PsA [14]. Clinical enthesitis affects up to at least a third of PsA patients, and the numbers may be higher as enthesitis is sometimes difficult to assess clinically, or patients may be asymptomatic. One study found that by using ultrasound imaging a greater number of psoriasis patients had entheseal abnormalities detected when compared with a control group [15]. These patients did not have clinical signs of PsA. The study concludes that psoriasis patients with subclinical enthesitis should be closely monitored for PsA development. The use of better imaging techniques have enabled clinicians to better recognise patients with enthesitis, and 
acknowledge that enthesitis may result in more diffuse inflammation than was initially thought [14]. Studies using animal models have shown arthritis originating from entheseal sites [14]. This process may be triggered by microtrauma and biomechanical stress which leads to an altered immune response and subsequent development of PsA [14].

An emerging new theory is the relationship between microbiome and inflammatory diseases. Advances in technology have enabled scientists to study the interactions between gut organisms and the development of autoimmunity. There is a known association between gut inflammation and infection and rheumatic diseases such as ankylosing spondylitis and reactive arthritis. Studies have shown that when rats with HLA B27 are grown under germ free conditions they fail to develop any gut or joint inflammation [16]. Research has also showed that PsA patients have lower levels of certain gut microbes (Akkermansia, Ruminococcus) when compared with patients with psoriasis and normal controls [16]. This lack of gut microbe diversity in PsA is also seen in inflammatory bowel disease [17]. Alterations in intestinal microbe diversity may cause an altered immune system leading to the development of inflammatory diseases, although further studies are needed in order confirm a causative relationship [17]. This work can provide clinicians with potential diagnostic aids and therapeutic strategies in the future, and further research questions can explore whether examining a patient's gut microbiome environment can help predict treatment response [16]. Clinicians could then develop more targeted treatment strategies for individual patients.

\section{Clinical Phenotypes}

PsA has a diverse range of clinical phenotypes which can make diagnosis challenging. Patients can present with an asymmetrical poly or oligoarthrits. Typically it is the distal interphalangeal (DIP) joints which are affected in comparison to RA where it is more metacarpophalangeal MCP and proximal interphalangeal PIP predominant joint disease. However PsA can also present with a symmetrical polyarthritis similar to RA. On average arthritis occurs 10 years after the development 
of psoriasis, but physicians should be aware that the arthritis can occur before development of skin lesions in around $15 \%$ of cases [1].

On some occasions patients may only have dactylitis as their presenting complaint. Axial disease can be an isolated feature in up to $5 \%$. Patients with PsO who complain of inflammatory back pain should be investigated further. Severe cases of PsA can result in arthritis mutilans, where the peripheral joints are deformed and shortened secondary to severe osteolysis. It should be noted that patients can present with a combination of these symptoms and the natural history of the disease may encompass any of these patterns depending on treatment and disease progression [6].

PsA is a chronic condition, which can progressively worsen if not adequately treated. Progressive joint damage can occur within the first 2 years of disease onset [2]. Polyarticular disease is the most frequent clinical manifestation [6] which slowly progresses. Factors which can predict a poor prognosis if present at presentation include polyarticular disease, female sex and raised inflammatory markers. Some studies have shown that patients with active disease at presentation who are treated with an intensive treat to target regime experienced better disease outcomes [18], although long term follow up data is needed to assess impact on structural damage. This model has yet to be adopted into standard clinical practice, but it could provide a direction for future treatment strategies to try and achieve better disease control and improve patients' quality of life. A validated, intensive treatment approach that is standardised could prevent progressive joint damage and avoid the significant healthcare and economic burdens that are caused by advanced PsA. In addition a biomarker for radiological progression would help target those patients who were more likely to progress. 
Dactylitis (see figure 1) is common in PsA patients with up to $50 \%$ of patients affected [6]. It is more common in the feet and specifically over the fourth toe. It is defined as fusiform swelling of the whole digit. Isolated dactylitis can be injected with steroid. Clinicians should be aware of differentials such as Gout, Sarcoid and infection [6]. The exact trigger behind dactylitis remains unclear. It is thought to be secondary to direct physical injury [19] although this hypothesis remains unproven. One study which examined the relationship between plantar forefoot pressures and dactylitis in PsA patients did not find a direct correlation between the two [19], but concluded that further research using larger sample sizes and more sophisticated measures were required to identify whether plantar pressure variations could result in dactylitis development in PsA patients.

Figure 1: Dactylitis of the second toe

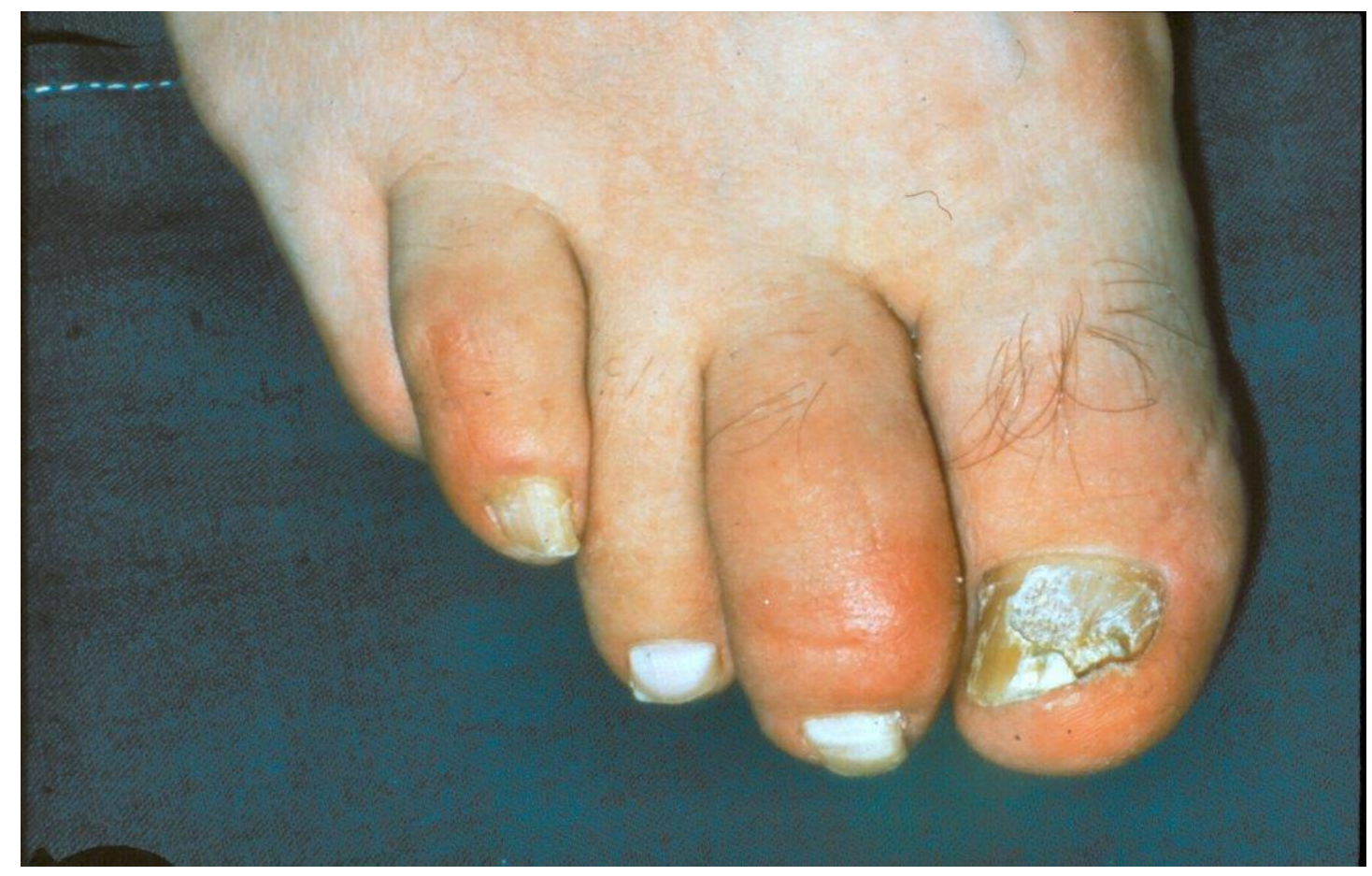


PsA patients can present with oligoarthritis or asymmetrical polyarthritis. DIP joints are commonly affected. However it can also present similarly to RA with a symmetrical pattern (see figure 2). Thus clinical assessment for patients with new onset inflammatory arthritis should include examination of skin especially over the elbows, knees, umbilicus, behind the ears and the natal cleft, where Pso lesions could be hidden [6]. The nails should also be examined for any onycholysis or pitting. A careful assessment of these sites can give vital clues as to the underlying cause of a patient's joint pain, and can help differentiate between PsA and differentials such as RA and osteoarthritis.

Figure 2: Symmetrical Polyarthritis

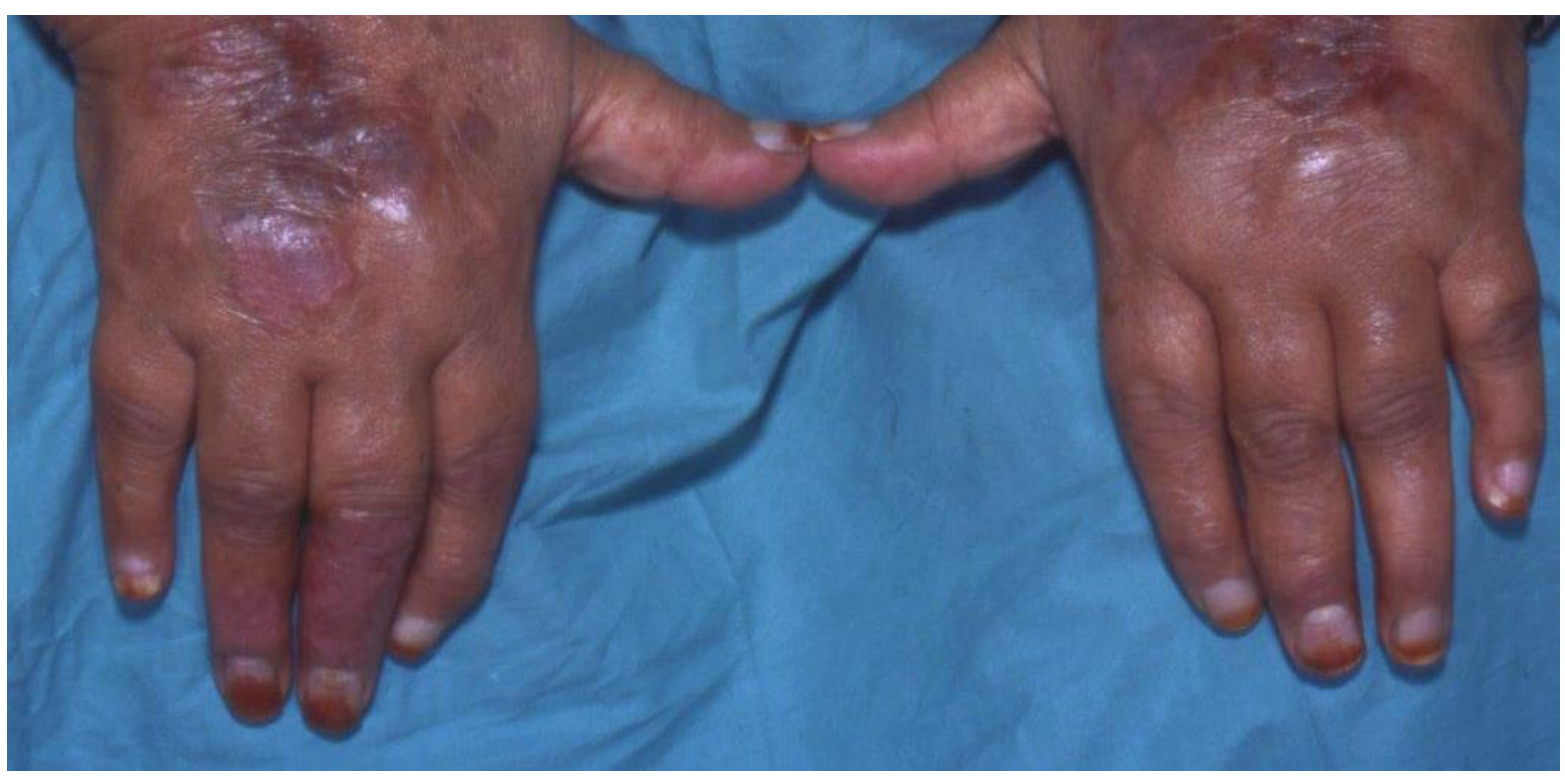




\section{Spinal involvement}

PsA patients may present with inflammatory sounding back pain, thus a good history is necessary to differentiate from mechanical causes. Inflammatory back pain tends to be worse with rest and improve with exercise. It may be nocturnal. There may be associated morning stiffness lasting longer than 30 minutes. Patients may complain of lower back pain which radiates into one or both buttocks. While the clinical presentation may be similar to ankylosing spondylitis, radiologically PsA more commonly displays asymmetrical sacroiliitis, whilst in AS it tends to be symmetrical. Some studies have also shown that AS patients had more severe axial involvement when compared with PsA [1].

\section{Enthesitis}

A hallmark of PsA, enthesitis can occur in up to a third of cases. It is characterised by inflammation affecting where a ligament or tendon attaches to the bone. One of the most common sites affected is the Achilles tendon. Enthesitis if present can be associated with greater overall disease burden [20]. A thorough assessment is required to identify enthesitis as it can be missed on routine clinical examination. Different indices have been developed to help assess and measure enthesitis, including the Leeds Enthesitis Index [21]. However there is no consensus yet on which measure should be accepted as standard [21].

Investigations

\section{Serum Biomarkers}

There are no serum biomarkers yet available that would predict development of PsA [22] in clinical practice, unlike in RA where rheumatoid factor and anti-cyclic citrullinated peptide are measured as part of the clinical assessment. In PsA these markers are negative in up to $95 \%$ of patients. CReactive Protein (CRP) can be measured to assess whether there is active inflammation in a patient with joint pains and is raised in $40-50 \%$ of patients $[1,6]$. If present it could indicate a more 
aggressive disease course and patients may have a more favourable response to disease modifying treatment. However CRP is not specific and it can be raised in other co-existing conditions such as infection. CRP levels can also increase with a patient's age. Up to $25 \%$ of patients may be positive for HLA-B27, which is present in other spondylarthropathy diseases [1].

There has been interest in developing a PsA biomarker to help facilitate early diagnosis especially for use in non-specialist settings given how challenging PsA can be to diagnose [12, 23]. One study has examined the use of urinary biomarkers to help distinguish between different inflammatory rheumatic diseases such as RA and PsA [23]. They were able to identify peptides which when detected in urine can help distinguish between each disease. The advantage of such a test is that it is simple to perform and can be readily available in primary care settings with the correct kit. The study was limited by a small sample size and further work is needed to validate these results. Nevertheless it is a promising area of research. There have been pilot studies which have found increased levels of soluble biomarkers such as matrix metalloproteinase-3 and osteoprotegerin in PsA patients when compared with patients with only psoriasis or healthy controls [24]. Developing biomarkers from other tissues such as skin and synovial fluid have also been investigated. A recent study found that a combination of three previously identified biomarkers (ITGB5, M2BP \& CRP) found in skin and synovial fluid in PsA patients were also elevated in the serum of PsA patients, and that a combination of these markers could help differentiate PsA from psoriasis, although the PsA cohort were patients with established disease [12]. The study also found that this combination performed better than CRP alone as a discriminator. Again, further trials from larger cohorts are needed to validate these results, but the development of biomarkers that can aid in diagnosis could help physicians identify PsA patients and start treatments earlier.

\section{Plain Radiographs}

Radiographs of the peripheral and axial joints can be helpful in diagnosis. Hallmark inflammatory findings such as erosions and joint space narrowing can be present [6]. More specific to PsA is new 
bone formation and enthesophytes. The 'pencil-in-cup' deformity is a typical feature ofPsA, appearances which result from periarticular erosions and bone resorption. Small joint pattern involvement in PsA tends to be more distal rather than the MCP joints. Osteoporosis tends to be uncommon in PsA when compared with RA. PSA involving the axial skeleton tends to result in unilateral sacroilitis in comparison to bilateral sacroilitis in Ankylosing Spondylitis. Syndesmophytes which are bony outgrowths which occur in both conditions may also be different in PsA as they may be paramarginal. Ligamentous calcification and joint ankylosis can also be features [6]. Traditionally plain films have been used to aid in diagnosis and monitor progression, but it should be noted that in early disease plain films may be normal, which could cause delay in diagnosis and treatment. Thus imaging modalities are needed which are more sensitive at detecting early or subclinical inflammation.

\section{Ultrasound}

Ultrasound is now being used in routine practice to detect inflammation in arthritis including PsA. It can be useful in detecting soft tissue inflammation and enthesitis which plain films and clinical examination are unable to accurately assess. Ultrasound is most often used to establish the underlying diagnosis but it is not routinely used to monitor progression. The use of ultrasound is very operator dependent, and there may also be confounders in distinguishing between PsA patients and normal controls such as obesity [24].

MRI

MRI can be used to recognise features of PsA such as enthesitis and spondylitis. It is a more sensitive method of assessing inflammation when compared with plain radiographs [25]. There has been interest in developing whole body MRI assessment scores for PsA given how the disease can affect different anatomical sites at the same time. Pilot studies have thus far shown that whole body MRI can identify inflammation in the spine and proximal peripheral joints but recognising more distal joint inflammation was more challenging, possibly due to lower image resolution being used [25]. 
With further studies and validation whole body MRI could offer a promising tool to assess and monitor disease progression in PsA patients.

\section{Classification \& Screening}

In theory PsA would seem a simple condition to diagnose as it would involve screening for the presence of inflammatory joint pains in the presence of psoriatic skin lesions. However joint inflammation in PsA can precede development of skin lesions in $15 \%$ of cases [2]. It can sometimes be challenging to differentiate between PsA and osteoarthritis especially in older patients as they both cause tenderness and swelling over the distal interphalangeal joints. Skin psoriasis may be subtle and affect areas not normally covered by clinical examination such as behind the ears and the natal cleft. These issues could result in delayed diagnosis and referral of PsA resulting in progressive joint damage for the patient. Even a 6 month delay in diagnosis could from initial presentation may result in a higher chance of developing erosive joint changes and lower quality of life scores for patients as shown by one study [26]. Thus there is a need for a specific and sensitive screening tool which can help identify and discriminate early PsA from other arthropathies - see below.

PsA was initially defined by Moll and Wright as 'an inflammatory arthritis in the presence of psoriasis with a usual absence of rheumatoid factor'. Since their pioneering work further understanding of PsA has enabled clinicians to develop classification criteria. PsA is part of the spondyloarthropathy (spa) group of diseases which include Reactive arthritis, Ankylosing Spondylitis, arthritis associated with inflammatory bowel disease and Juvenile arthritis. These conditions share similar clinical characteristics and are associated with HLA B27. As a consequence some criteria have developed which help diagnose $\mathrm{SpA}$ as one group [27].

Including PsA in a standardised classification criteria for SpA can be difficult given the myriad clinical phenotypes with which PsA can present. Criteria have been developed which take into account the 
varied presentations. The Classification of Psoriatic Arthritis (CASPAR) criteria (table 1) were developed after comparing existing criteria [28] and were developed on a large database of patients with PSA $(n=588), R A(n=384)$ and AS $(n=72)$. CASPAR was primarily designed for use in epidemiological research settings, but can also be applied to clinic settings [29]. CASPAR is highly specific for PsA at $99.1 \%$ but not as sensitive at $87.4 \%$ [22], although studies have found it more sensitive when compared with other criteria [29]. CASPAR's advantage is that it is simple to apply even by non-specialists but a problem remains with the stem of 'inflammatory articular disease' which needs further definition. A patient who scores 3 or more on the criteria should be referred for further specialist assessment.

Table 1 CASPAR criteria: can be applied to anyone with inflammatory musculoskeletal disease (joints, entheses or spine).

\begin{tabular}{ll}
\hline Criteria & Score \\
\hline Skin Psoriasis & 2 \\
Present? & 1 \\
Previous history? (if patient unaffected) & 1 \\
$\quad$ Family history? (if patient unaffected) & 1 \\
Nail lesions & 1 \\
Dactylitis or history of dactylitis & 1 \\
Rheumatoid factor negative & 1 \\
Juxta-articular new bone formation on radiograph & \\
\hline
\end{tabular}

Screening tools have been developed such as the Psoriasis Epidemiology Screening Tool (PEST) and the Psoriasis and Arthritis Screening Questionnaire (PASQ). These can be used in clinics and primary care settings to help identify at risk psoriasis patients [22]. Identifying patients early means clinicians can start treatment before any joint damage develops [22]. However clinicians should be aware that 
some studies have shown that these questionnaires also identify other musculoskeletal disorders and not just PsA [30]. Therefore they lack discriminatory value and may not pick up subsets of PsA patients [30]. Further work is needed to improve the specificity of these tools.

\section{Current Treatment}

Treating PsA is challenging given the varying clinical phenotypes patients. An ideal therapeutic option would be one which can effectively treat all disease domains, especially controlling both skin and joint manifestations. There is a paucity of evidence concerning traditional disease modifying anti-rheumatic drug use in PsA when compared with RA.

Research is being conducted into developing new and effective treatments for PsA. Despite advances in therapeutics, there remains an unmet need in developing an optimal treatment for PsA. One study which examined physician and patient perspectives in the management of psoriasis and PsA found that many clinicians found treating these conditions complicated and challenging [5]. Many patients have expressed dissatisfaction and frustration with their current treatment [4]. This study shows that there is a need to develop more effective and less burdensome therapies for PsA.

\section{ANTI-INFLAMMATORIES/CORTICOSTEROIDS}

In patients who present with mild oligoarticular disease, non-steroidal anti-inflammatories (NSAIDs) can be used to manage symptoms. If a specific joint is painful and swollen a local corticosteroid injection into that joint can help with local inflammation. Patients with enthesitis can also be treated with local steroid injections, although there is a high risk of Achilles tendon rupture with a local steroid injection around the insertion - injection into the retrocalcaneal bursa is acceptable. A patient suffering from a PsA flare could benefit from an intramuscular depomedrone injection, but patients should be warned about the risk of skin psoriasis flare with this injection. Despite the theoretical risk of skin flares corticosteroids are used in the treatment of PsA for their rapid anti- 
DMARDS

Evidence for the use of traditional DMARDs use remains limited [1]. Most clinicians would use methotrexate as their first line agent, given their familiarity with the drug. Studies however have shown mixed results [3]. One study found no difference when compared with placebo, but this may have been secondary to using a lower than standard dose [3]. There have been other studies which have shown that methotrexate has had at least a moderate effect on improving PsA, although it should be noted that there are no data suggesting that it has a beneficial effect on radiographic progression [2]. Better designed studies are needed to validate its use in PsA. There have been studies which have shown a beneficial effect with leflunomide for both peripheral arthritis and skin lesions [2]. There has been little recent data on the use of Sulphasalazine [32], but a Cochrane review suggested a small beneficial effect for peripheral synovitis. Its effect on radiological progression is unknown [2]. DMARDs do not seem to have any effect on other domains of PsA such as entheistis, although leflunomide may improve dactylitis [32]. Data on the use of combination DMARD therapy in PsA is also limited. Many treatment recommendations would advise starting with DMARD therapy in PsA patients but clinicians should be aware that there may be limited therapeutic effects for most domains of the disease. Their use can also be limited by their side effect profile such as liver toxicity, particularly in patients who have co-morbidities such as fatty liver disease [2].

BIOLOGIC THERAPY

Anti-TNF therapy 
The use of Tumour Necrosis Factor-inhibitor drugs (TNF-i) have helped to improve the management of PsA patients. These agents are effective in both PsA and psoriasis, are clinically superior to traditional DMARDS and have been shown to inhibit radiographic progression [1]. Evidence also shows that they can treat other PsA domains such as enthesitis and axial disease [1]. Patients would have to fail a trial of DMARD therapy before they could be considered eligible for biologics according to most treatment guidelines largely due to cost issues surrounding the drugs. Once established on therapy patients have to be monitored for adverse effects such as congestive cardiac failure, immunosuppression, demyelination and drug induced lupus. As with DMARDs, patients require routine blood monitoring. There have been studies which have shown patients dissatisfaction with such treatment regimens either due to adverse effects or the intensive monitoring which is required $[3,4]$. Some patients may also struggle with self-administering TNF-i injections due to hand pain and deformities secondary to PsA. There may also be fear of needles leading to some patients becoming non-compliant with treatment [4]. Physicians can find initiating biologics burdensome due to the time needed for patient education and counselling as well as cost of the drugs [5].

\section{Interleukin inhibitors}

There has been a lack of therapeutic options for PsA patients who fail both DMARD and TNF-i in the past. Drugs such as Rituximab and Tociluzimab which are highly effective for RA have only shown modest benefits for PsA patients thus far in trials [3]. There is also a need to develop therapies which can treat all domains of the disease with better tolerability and less need for regular monitoring. Recent advances in therapeutics driven by these issues have enabled clinicians to have more options for treatment if patients fail first and second line therapies. Drugs such as Ustekinumab (an interleukin (IL)-12/23 blocker) and Secukinumab (an IL-17A blocker) have been found to beneficial for both skin and joints and have been reasonably well tolerated in clinical trials [3]. Data for Ustekinumab has shown efficacy for enthesitis and dactylitis [3]. Like TNF-i these drugs are only 
currently available as injections so some patients could struggle to use them. Secukinumab can also cause recurrent candida infections as an adverse effect.

\section{Phosphodiesterase 4 inhibitors}

Apremilast is an oral agent which acts as an inhibitor of the enzyme phosphodiesterase 4, which in turn leads to elevated levels of cyclic adenosine monophosphate (cAMP). This results in down regulation of pro-inflammatory cytokines [3]. Trials have demonstrated that Apremilast is effective for PsA when compared with placebo, and was well tolerated with no laboratory monitoring required $[3,33]$. Its effect on skin response seems to be comparable to methotrexate [1]. Apremilast may better tolerated by some patients when compared with TNF-i as it is an oral drug with minimal side effects, although some studies have shown that joint response is lower in biologic experienced patients in comparison to biologic naïve patients [33]. Adverse effects include diarrhoea, upper respiratory tract infections and weight loss [33].

\section{Janus Kinase inhibitors}

Janus kinase inhibitors have been shown to be beneficial in psoriasis, with improvement in Psoriasis Area and Severity Index scores (PASI) demonstrated in clinical trials [34]. Janus kinase are involved in cytokine signalling. Tofacitinib is an oral janus kinase inhibitor which is currently being investigated for use in PsA, with some early studies showing efficacy of Tofacitinib in PsA with a manageable safety profile [35], although further research with larger sample sizes are needed to validate its efficacy.

T cell modulation

Abatacept has been used to effectively treat RA patients and works by inhibiting CTLA- 4 which in turn causes down regulation of T cells [3]. There have been some studies which have shown an 
improvement in PsA patients on Abatacept when compared with placebo, although the skin response was more modest [36]. Again the greatest effect was seen in TNF-i naïve patients.

Further studies are needed in order to validate these agents, in particular more head to head studies comparing efficacy with traditional DMARDS and biologic therapy in order to better stratify treatment choices. Treatment strategies are also needed for patients who may present with predominant oligoarthritis or enthesitis.

Unmet needs

The following table summarises the unmet needs in PsA which have been identified by this review, and what the implications could be for patient care if these needs are addressed in future studies.

Table 2: Summary of unmet needs in PsA

\begin{tabular}{|l|l|l|l|}
\hline Domain & Unmet need & Current position & Implication \\
\hline Genetics & Are there any & HLA subtypes & Clinicians could \\
& $\begin{array}{l}\text { genetic markers for } \\
\text { severity or treatment } \\
\text { response? }\end{array}$ & including $B^{*} 27: 05: 02$, & use genetic \\
& $B^{*} 08: 01: 01-$ & information for \\
& linked to individual & targeted \\
& phenotypes in PsA & treatment \\
& & & \\
\hline
\end{tabular}




\begin{tabular}{|c|c|c|c|}
\hline Triggers & $\begin{array}{l}\text { What is the exact } \\
\text { pathogenic trigger } \\
\text { behind the } \\
\text { development of PsA? }\end{array}$ & $\begin{array}{l}\text { Role of microbiome in } \\
\text { triggering PsA }\end{array}$ & $\begin{array}{l}\text { Microbiome } \\
\text { might provide } \\
\text { therapeutic } \\
\text { target }\end{array}$ \\
\hline Biomarkers & $\begin{array}{l}\text { Are there any } \\
\text { biomarkers to aid } \\
\text { diagnosis, predict } \\
\text { severity or treatment } \\
\text { response? }\end{array}$ & $\begin{array}{l}\text { Potential serum } \\
\text { biomarkers include } \\
\text { ITGB5, M2BP \& CRP. } \\
\text { Urinary biomarkers } \\
\text { could identify } \\
\text { inflammatory articular } \\
\text { disease }\end{array}$ & $\begin{array}{l}\text { Can be used by } \\
\text { non-specialists to } \\
\text { aid in diagnosis }\end{array}$ \\
\hline Screening & $\begin{array}{l}\text { Are there any } \\
\text { tools which are } \\
\text { both highly } \\
\text { specific and } \\
\text { sensitive for } \\
\text { detecting early } \\
\text { PsA? }\end{array}$ & $\begin{array}{l}\text { Current } \\
\text { questionnaires ie PEST } \\
\text { have low specificity }\end{array}$ & $\begin{array}{l}\text { Earlier diagnosis } \\
\text { and better } \\
\text { prognosis }\end{array}$ \\
\hline
\end{tabular}




\begin{tabular}{|c|c|c|c|}
\hline Screening & $\begin{array}{l}\text { Can pre-disease } \\
\text { be identified by } \\
\text { imaging? }\end{array}$ & $\begin{array}{l}\text { Ultrasound identifies } \\
\text { enthesial } \\
\text { abnormalities but } \\
\text { exact implications of } \\
\text { this remains unclear }\end{array}$ & $\begin{array}{l}\text { Earlier diagnosis } \\
\text { and better } \\
\text { prognosis }\end{array}$ \\
\hline Diagnosis & $\begin{array}{l}\text { Recognition of } \\
\text { inflammatory } \\
\text { musculoskeletal } \\
\text { disease in } \\
\text { application of } \\
\text { CASPAR criteria }\end{array}$ & $\begin{array}{l}\text { No reliable clinical } \\
\text { scoring system }\end{array}$ & $\begin{array}{l}\text { Easier application } \\
\text { of CASPAR in } \\
\text { studies and in } \\
\text { clinical practice }\end{array}$ \\
\hline Dactylitis & $\begin{array}{l}\text { What is the } \\
\text { trigger behind } \\
\text { dactylitis? }\end{array}$ & $\begin{array}{l}\text { Theory that trauma } \\
\text { may lead to dactylitis } \\
\text { (deep Koebner } \\
\text { phenomenon) } \\
\text { No evidence that } \\
\text { plantar pressure } \\
\text { important }\end{array}$ & $\begin{array}{l}\text { Reduction of risk } \\
\text { of trauma may } \\
\text { reduce dactylitis } \\
\text { prevalence }\end{array}$ \\
\hline Enthesitis & $\begin{array}{l}\text { No reliable } \\
\text { clinical indices }\end{array}$ & $\begin{array}{l}\text { Current indices have } \\
\text { poor correlation to } \\
\text { imaging enthesitis } \\
\text { except at the Achilles }\end{array}$ & $\begin{array}{l}\text { More reliable } \\
\text { outcome } \\
\text { measurements } \\
\text { for entheistis }\end{array}$ \\
\hline
\end{tabular}




\begin{tabular}{|c|c|c|c|}
\hline Treatment & $\begin{array}{l}\text { Are current } \\
\text { treatment } \\
\text { strategies } \\
\text { adequate? }\end{array}$ & $\begin{array}{l}\text { TICOPA completed, } \\
\text { AMGEN underway, } \\
\text { other studies on drug } \\
\text { sequencing i.e which } \\
\text { class of drug for first } \\
\text { biologic }\end{array}$ & $\begin{array}{l}\text { Updated } \\
\text { treatment } \\
\text { guidelines }\end{array}$ \\
\hline Treatment & $\begin{array}{l}\text { Does early } \\
\text { intensive } \\
\text { treatment } \\
\text { improve } \\
\text { outcomes? }\end{array}$ & $\begin{array}{l}\text { TICOPA improved } \\
\text { outcomes at } 12 \\
\text { months but long term } \\
\text { unknown } \\
\text { Observational data } \\
\text { suggests that earlier } \\
\text { treatment improves } \\
\text { long term outcomes }\end{array}$ & Better outcomes \\
\hline Treatment & $\begin{array}{l}\text { Are systemic } \\
\text { steroids (intra- } \\
\text { articular and } \\
\text { intramuscular) } \\
\text { effective without } \\
\text { causing skin } \\
\text { flare? }\end{array}$ & $\begin{array}{l}\text { Anecdotal reports of } \\
\text { skin flare with the use } \\
\text { of oral steroids } \\
\text { TICOPA demonstrated } \\
\text { small numbers of } \\
\text { individual flares but } \\
\text { no group differences }\end{array}$ & $\begin{array}{l}\text { Evidence for use } \\
\text { of this class of } \\
\text { drugs }\end{array}$ \\
\hline
\end{tabular}




\begin{tabular}{|c|c|c|c|}
\hline Treatment & $\begin{array}{l}\text { Lack of evidence } \\
\text { for conventional } \\
\text { synthetic } \\
\text { DMARDs }\end{array}$ & $\begin{array}{l}\text { Weak evidence for } \\
\text { Sulphaslazine \& } \\
\text { Methotrexate, only } \\
\text { one study for } \\
\text { Leflunomide }\end{array}$ & $\begin{array}{l}\text { Evidence for use } \\
\text { of this class of } \\
\text { drugs }\end{array}$ \\
\hline Treatment & $\begin{array}{l}\text { Are there any } \\
\text { advances in } \\
\text { treatment which } \\
\text { could be applied } \\
\text { to PsA? }\end{array}$ & $\begin{array}{l}\text { Targets IL-23, IL-22, } \\
\text { JAK, PDE4 }\end{array}$ & $\begin{array}{l}\text { Additional } \\
\text { therapeutic } \\
\text { options available } \\
\text { if treatment } \\
\text { failure or } \\
\text { intolerance to } \\
\text { traditional } \\
\text { therapies }\end{array}$ \\
\hline
\end{tabular}

\section{Conclusions}

There have been significant advances made in our understanding of PsA which has led to better patient care. Yet the disease can still result in disability, pain, reduced quality of life and depression for many patients. Delayed diagnosis coupled with burdensome treatment has meant that the care of PsA can still be considered sub-optimal especially when compared with RA. Advances in understanding the pathogenic mechanisms underlying PsA will enable physicians to better identify early PsA cases and tailor treatments specifically for individual patients, allowing for more favourable outcomes and less issues of tolerability and safety. 
[1] Ritchlin CT, Colbert RA, Gladman D. Psoriatic Arthritis. N Engl J Med 2017; 376:957-970

[2] Mease PJ, Armstrong AW. Managing Patients with Psoriatic Disease: The Diagnosis and Pharmacologic Treatment of Psoriatic Arthritis in Patients with Psoriasis. Drugs. 2014;74(4):423-441.

[3] DoQuyen Huynh, Arthur Kavanaugh; Psoriatic arthritis: current therapy and future approaches. Rheumatology (Oxford) 2015; 54 (1): 20-28.

[4] Kavanaugh A, Helliwell P, Ritchlin CT. Psoriatic Arthritis and Burden of Disease: Patient Perspectives from the Population-Based Multinational Assessment of Psoriasis and Psoriatic Arthritis (MAPP) Survey. Rheumatology and Therapy. 2016;3(1):91-102.

[5] Van de Kerkhof PCM, Reich K, Kavanaugh A, et al. Physician perspectives in the management of psoriasis and psoriatic arthritis: results from the population-based Multinational Assessment of Psoriasis and Psoriatic Arthritis survey. Journal of the European Academy of Dermatology and Venereology. 2015;29(10):2002-2010.

[6] Coates LC, Helliwell P. Psoriatic Arthritis. Oxford Textbook of Rheumatology $4^{\text {th }}$ edition 2016 Chapter 114

[7] Nograles KE, Brasington RD, Bowcock AM. New insights into the pathogenesis and genetics of psoriatic arthritis. Nature clinical practice Rheumatology. 2009;5(2):83-91.

[8] Busse K, Liao W. Which Psoriasis Patients Develop Psoriatic Arthritis? Psoriasis forum / National Psoriasis Foundation. 2010;16(4):17-25.

[9] Palazzi C, et al. Hepatitis C virus infection in psoriatic arthritis. Arthritis Rheum. 2005;53:223-5.

[10] FitzGerald O, Haroon M, Giles JT, Winchester R. Concepts of pathogenesis in psoriatic arthritis: genotype determines clinical phenotype. Arthritis Research \& Therapy. 2015;17(1):115.

[11] Haroon M, Winchester R, Giles JT, et al Certain class I HLA alleles and haplotypes implicated in susceptibility play a role in determining specific features of the psoriatic arthritis phenotype Annals of the Rheumatic Diseases 2016;75:155-162 
[12] Cretu, D., Gao, L., Liang, K., Soosaipillai, A., Diamandis, E. P. and Chandran, V. (), Novel serum biomarkers differentiate psoriatic arthritis from psoriasis without psoriatic arthritis. Arthritis Care \& Research 2017. Accepted Author Manuscript [epub ahead of print]

[13] Kerschbaumer A, Fenzl KH, Erlacher L, Aletaha D. An overview of psoriatic arthritis epidemiology, clinical features, pathophysiology and novel treatment targets. Wiener Klinische Wochenschrift. 2016;128(21):791-795.

[14] McGonagle, D. Enthesitis: an autoinflammatory lesion linking nail and joint involvement in psoriatic disease. Journal of the European Academy of Dermatology and Venereology, 2009 23: 9-13.

[15] Gisondi P, Tinazzi I, El-Dalati G, et al Lower limb enthesopathy in patients with psoriasis without clinical signs of arthropathy: a hospital-based case-control study Annals of the Rheumatic Diseases 2008;67:26-30

[16] Gill T, Asquith M, Rosenbaum JT, Colbert RA. The Intestinal Microbiome in Spondyloarthritis. Current opinion in rheumatology. 2015; 27(4):319-325.

[17] Scher JU, Ubeda C, Artacho A, et al. Decreased Bacterial Diversity Characterizes an Altered Gut Microbiota in Psoriatic Arthritis and Resembles Dysbiosis of Inflammatory Bowel Disease. Arthritis \& rheumatology (Hoboken, NJ). 2015;67(1):128-139. doi:10.1002/art.38892.

[18] Coates LC, Moverley AR, McParland L, et al. Effect of tight control of inflammation in early psoriatic arthritis (TICOPA): a UK multicentre, open-label, randomised, controlled trial. Lancet (London, England). 2015;386(10012):2489-2498.

[19] Wilkins RA, Siddle HJ, Redmond AC, Helliwell PS. Plantar forefoot pressures in psoriatic arthritisrelated dactylitis: an exploratory study. Clinical Rheumatology. 2016;35:2333-2338.

[20] Mease, P. J., Karki, C., Palmer, J, et al. Clinical Characteristics, Disease Activity, and Patient-Reported Outcomes in Psoriatic Arthritis Patients with Dactylitis or 
Enthesitis: Results from the Corrona Psoriatic Arthritis/Spondyloarthritis Registry. Arthritis Care \& Research. Accepted Author Manuscript. 2017

[21] Mease PJ, Van den Bosch F, Sieper J, et al. Performance of 3 Enthesitis Indices in Patients with Peripheral Spondyloarthritis During Treatment with Adalimumab. J Rheumatol. 2011 Aug; 38(8):1656-60.

[22] Raychaudhuri SP, Wilken R, Sukhov AC, et al. Management of psoriatic arthritis: Early diagnosis, monitoring of disease severity and cutting edge therapies, Journal of Autoimmunity, Volume 76, 2017, Pages 21-37.

[23] Siebert, S. et al. Urinary proteomics can define distinct diagnostic inflammatory arthritis subgroups. Sci. Rep. 2017 7, 40473.

[24] Gladman DD. Recent advances in understanding and managing psoriatic arthritis. F1000Research. 2016;5:2670.

[25] René Panduro Poggenborg, Susanne Juhl Pedersen, Iris Eshed, Inge Juul Sørensen, Jakob M. Møller, Ole Rintek Madsen, Henrik S. Thomsen, Mikkel Østergaard; Head-to-toe whole-body MRI in psoriatic arthritis, axial spondyloarthritis and healthy subjects: first steps towards global inflammation and damage scores of peripheral and axial joints. Rheumatology (Oxford) 2015; 54 (6): 1039-1049.

[26] Haroon M, Gallagher P, FitzGerald O: Diagnostic delay of more than 6 months contributes to poor radiographic and functional outcome in psoriatic arthritis. Ann Rheum Dis. 2015;74(6):1045-50. 10.1136/annrheumdis-2013-204858

[27] Rudwaleit M, van der Heijde D, Landewé R, et al. The Assessment of SpondyloArthritis international Society classification criteria for peripheral spondyloarthritis and for spondyloarthritis in general. Annals of the Rheumatic Diseases 2011;70:25-31 
[28] Taylor, W., Gladman, D., Helliwell, P, et al (2006), Classification criteria for psoriatic arthritis: Development of new criteria from a large international study. Arthritis \& Rheumatism, 54: 2665-2673.

[29] Congi L, Roussou E. Clinical application of the CASPAR criteria for psoriatic arthritis compared to other existing criteria. Clin Exp Rheumatol. 2010 May-Jun;28(3):304-10.

[30] Coates, L.C., Aslam, T., Al Balushi, F, et al. Comparison of three screening tools to detect psoriatic arthritis in patients with psoriasis (CONTEST study). British Journal of Dermatology, 2013 168: 802-807

[31] Coates, L.C. and Helliwell, P.S. Psoriasis flare with corticosteroid use in psoriatic arthritis. Br J Dermatol. 2016 174: 219-221.

[32] Marchesoni A, Lubrano E, Cauli A et al. Psoriatic Disease: Update on Traditional Diseasemodifying Antirheumatic Drugs. J Rheumatol Suppl. 2015 Nov; 93:61-4.

[33] Kavanaugh A, Mease PJ, Gomez-Reino JJ, et al. Treatment of psoriatic arthritis in a phase 3 randomised, placebo-controlled trial with apremilast, an oral phosphodiesterase 4 inhibitor. Annals of the Rheumatic Diseases. 2014;73(6):1020-1026.

[34] Elyoussfi S, Thomas BJ, Ciurtin C. Tailored treatment options for patients with psoriatic arthritis and psoriasis: review of established and new biologic and small molecule therapies. Rheumatology International. 2016;36:603-612.

[35] Asahina A, Etoh T, Igarashi A, et al. Oral tofacitinib efficacy, safety and tolerability in Japanese patients with moderate to severe plaque psoriasis and psoriatic arthritis: A randomized, double-blind, phase 3 study. The Journal of Dermatology. 2016;43(8):869-880.

[36] Mease PJ, Gottlieb AB, van der Heijde D, et al Efficacy and safety of abatacept, a T-cell modulator, in a randomised, double-blind, placebo-controlled, phase III study in psoriatic arthritis Annals of the Rheumatic Diseases Published Online First: 04 May 2017. 
